\title{
Comment on "How effective and efficient are multiobjective evolutionary algorithms at hydrologic model calibration?" by Y. Tang et al., Hydrol. Earth Syst. Sci., 10, 289-307, 2006
}

\author{
J. A. Vrugt \\ Center for NonLinear Studies (CNLS), Los Alamos National Laboratory, Los Alamos, NM 87545, USA
}

Received: 10 January 2007 - Published in Hydrol. Earth Syst. Sci. Discuss.: 31 January 2007

Revised: 20 June 2007 - Accepted: 24 June 2007 - Published: 10 July 2007

In a recent paper by Tang, Reed and Wagener (2006, hereafter referred to as TRW) a comparison assessment was presented of three state-of-the-art evolutionary algorithms for multiobjective calibration of hydrologic models. Through three illustrative case studies, TRW demonstrate that the Strength Pareto Evolutionary Algorithm 2 (SPEA2) and Epsilon Dominance Nondominated Sorted Genetic Algorithm ( $\varepsilon$-NSGAII) achieve a better performance than the Multiobjective Shuffled Complex Evolution Metropolis algorithm (MOSCEM-UA), previously developed by us and presented in Vrugt et al. (2003). I would like to congratulate TRW with their paper, which I believe provides a strong and valuable contribution to the field of hydrologic model calibration. However, I wish to differ in opinion about some of the main conclusions presented in their paper, especially with respect to the seemingly inferior performance of the MOSCEM-UA algorithm.

The results presented in TRW were obtained using uniform random sampling of the initial parameter space. Such a sampling strategy is widely used within the water resources and computational science literature, and expresses a situation where very little prior information is available about the location of the Pareto optimal solution set. The initial sample is subsequently iteratively improved using the various algorithmic steps in the employed evolutionary algorithm. It is however possible to significantly improve the efficiency and robustness of evolutionary search for case studies (2) and (3) reported in TRW if we first attempt to create an initial sample that approximates the Pareto tradeoff surface as closely as possible. In our original paper (Vrugt et al., 2003) we suggest such an alternative sampling strategy by first locating the theoretical ends of the Pareto set using classical single objective optimization, and to use traditional first-order statistical theory around the optimal estimators for these individual ob-

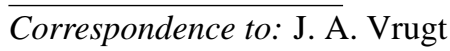

(vrugt@lanl.gov) jectives to create the initial sample of points. The results presented in Vrugt et al. (2003) have demonstrated that this alternative search strategy provides a computational efficient and robust alternative to multiobjective optimization.

It is surprising however that TRW have not considered this second or alternative optimization strategy in their paper. I believe that this alternative sampling approach should have been used in conjunction with the various algorithms, to appropriately disseminate and implement the ideas presented in Vrugt et al. (2003). If correctly used, this alternative search strategy would have provided the entire Pareto tradeoff surface as depicted in Fig. 5, at far less computational costs than the SPEA2 and $\varepsilon$-NSGAII algorithms. For example, preliminary analyses of the identification problem discussed in Fig. 5, suggest that state-of-the-art single objective search algorithms can identify the single criterion solutions of $\operatorname{RMSE}(R)$ and $R M S E(T)$ in less than 20000 function evaluations. Experience further suggests that about 2000 additional function evaluations would have been needed to sample the entire Pareto front using this prior information. This is considerably less than the 15000000 number of SAC-SMA model evaluations used to construct the results presented in TRW. Thus, in practice, this alternative search strategy using prior information from the single criterion ends of the Pareto front would have consistently received superior performance to the SPEA2 and $\varepsilon$-NSGAII algorithms. This would especially be true for the hydrologic model calibration problems, discussed in case study (2) and (3).

Nevertheless, the work presented in TRW addresses a number of critical issues related to the use of multiobjective optimization for the calibration of hydrologic models, and highlights the strengths and weaknesses of current available evolutionary search algorithms. In response to this, we (Vrugt and Robinson, 2007) have recently developed a new method called A Multi-ALgorithm Genetically Adaptive Multiobjective or AMALGAM method, that combines two new concepts, simultaneous multi-method search,

Published by Copernicus Publications on behalf of the European Geosciences Union. 
and self-adaptive offspring creation, to ensure a fast and computationally efficient solution to multiobjective optimization problems. By running a diverse set of optimization algorithms simultaneously for population evolution and adaptively favoring individual algorithms that exhibit the highest reproductive success during the search, AMALGAM has the ability to quickly adapt to the specific difficulties and peculiarities of the optimization problem at hand. Experiments conducted using standard, synthetic multi-objective test problems have shown that the AMALGAM method is on the order of 3-10 times more efficient than the SPEA2 and $\varepsilon$-NSGAII multiobjective optimization algorithms, and provides a final population that closely approximates the Pareto solution space. This new search method is relatively easy to implement, and is designed to take full advantage of the power of distributed computer networks (for an example implementation using OCTAVE/MPI see Vrugt et al., 2006). Recently, we have also developed a single objective optimization version of AMALGAM, called AMALGAM-SO that implements the concept of self-adaptive multimethod search within a restart strategy with increasing population size (Vrugt et al., $2007^{1}$ ). The results of this research will be reported in due course.
Acknowledgements. The author is supported by a J. Robert Oppenheimer Fellowship from the Los Alamos Postdoctoral Program.

Edited by: H. H. G. Savenije

\section{References}

Tang, Y., Reed, P., and Wagener, T.: How effective and efficient are multiobjective evolutionary algorithms at hydrologic model calibration?, Hydrol. Earth Syst. Sci., 10, 289-307, 2006, http://www.hydrol-earth-syst-sci.net/10/289/2006/.

Vrugt, J. A., Gupta, H. V., Bastidas, L. A., Bouten, W., and Sorooshian, S.: Effective and efficient algorithm for multiobjective optimization of hydrologic models, Water Resour. Res., 39(8), 1214, doi:10.1029/2002WR001746, 2003.

Vrugt, J. A., Nualláin, B. Ó, Robinson, B. A., Bouten, W., Dekker, S. C., and Sloot, P. M. A.: Application of parallel computing to stochastic parameter estimation in environmental models, Comput. Geosci., 32(8), 1139-1155, doi:10.1016/j.cageo.2005.10.015, 2006.

Vrugt, J. A. and Robinson, B. A.: Improved evolutionary search from genetically adaptive multi-method search, P. Natl. Acad. Sci. USA, 104(3), 708-711, doi:10.1073/pnas.0610471104, 2007.

\footnotetext{
${ }^{1}$ Vrugt, J. A., Robinson, B. A., and Hyman, J. M.: A universal multimethod search strategy for computationally efficient global optimization, IEEE T. Evolut. Comput., in review, 2007.
} 